\title{
Factors Influencing Stereotaxic Pulmonary Vein Isolation
}

\author{
Jean-Baptiste Guichard ${ }^{1 *}$, Antoine Da Costa ${ }^{1}$, Nicolas Maillard ${ }^{2}$, Cécile Romeyer ${ }^{1}$, Pierre Croisille ${ }^{3}$ and Karl Isaaz ${ }^{1}$ \\ ${ }^{1}$ Department of Cardiology, University Hospital of Saint-Étienne, University Jean Monnet, Saint-Étienne, France
}

${ }^{2}$ Department of Nephrology, University Hospital of Saint-Étienne, University Jean Monnet, Saint-Étienne, France

${ }^{3}$ Department of Radiology, University Hospital of Saint-Étienne, University Jean Monnet, Saint-Étienne, France

*Corresponding author: Dr Guichard JB, University Hospital of Saint-Etienne, Avenue Albert Raimond, 42000 Saint-Etienne, France

\begin{abstract}
Background - Catheter ablation of atrial fibrillation (AF) is performed to restore and maintain a sinus rhythm. Remote magnetic navigation system (RMNS) allows an efficient and safe procedure. Left atrial (LA) anatomic barriers of this device are not well known. Aims - This study was aimed to evaluate clinical, echocardiographic and cardiac computed tomography (CCT) anatomic LA characteristics as predictors of stereotaxic AF procedure duration. Methods - From February 2015 to April 2016, 102 symptomatic and drug refractory AF patients were consecutively enrolled in an observational, prospective trial when first $\mathrm{AF}$ ablation. $\mathrm{AF}$ Radiofrequency (RF) was performed with a RMNS using Niobe ${ }^{\circledR}$ ES. Clinical endpoints and LA characteristics were reported, prospectively by a transthoracic and transesophageal echocardiography, and CCT scan. Results - Mean patient age was $59 \pm 12$ years old, $77 \%$ male, mean CHA2DS2VASc of $1.3 \pm 1.3$ and mean LA surface of $23 \pm 6.5 \mathrm{~cm} 2$. Procedure duration of $97.2 \pm 32.9$ minutes and fluoroscopy duration of $13.4 \pm 7.9$ minutes were recorded. Persistent versus paroxysmal AF $(p<0.05)$, previous flutter ablation $(p<0.01)$, LA dilation $(p<0.05)$, narrow LA ridge $(p=0.01)$, small surface area and high eccentricity of the left inferior pulmonary vein (LIPV) $(\mathrm{p}<0.01)$ are correlated to an increased procedure duration. Previous flutter ablation $(\mathrm{p}<0.01)$, persistent AF ( $<<0.05), \mathrm{LIPV}$ eccentricity $(\mathrm{p}<0.05)$ and ridge width $(\mathrm{p}=0.05)$ were found to be independently associated with procedure duration. Conclusion - Our study is the first analyzing predictors of stereotaxic procedure duration. Narrow LA ridge, small and flattened LIPV were independently correlated with an increased procedure duration. Yet neither co-morbidity nor cardiomyopathy was associated to procedure changes.
\end{abstract}

Keywords: Atrial fibrillation ablation; pulmonary vein isolation; remote magnetic navigation; procedure duration; anatomic characteristics

\section{Introduction}

Radiofrequency ablation (RF) is a treatment of choice for atrial fibrillation (AF) because of a positive risk/benefit ratio compared to antiarrhythmic drugs [1-3].

Even if significant advances have been made over the past years regarding RF, pulmonary vein isolation (PVI) notably, several limitations remain to be overcome, such as the management of recurrences usually due to pulmonary veins (PV) reconduction, the high level of X-ray exposure and a significant risk of complications [4-6].

AF ablation is carried out in expert centers with high patient volumes. It is one of the most common procedures in electrophysiology departments (30 to $50 \%$ of total procedures) due to its prevalence and the recent guidelines [1-3]. The management of end cavity ablation in challenging clinical settings may lead to tedious and risky procedures [6,7]. and the evolvement of $\mathrm{AF}$ ablation indications lead to increased procedures per operator [8]. Consequently, operators are facing an increased X-ray exposition, fatigue and lack of concentration [4-6], leading to extended procedures and an increased complications risk $[6,9]$.

Recently, a remote magnetic navigation system (RMNS) was introduced as a way to ensure stable catheter positioning, to provide adequate tissue contact, and to reduce patient and physician X-ray exposure [10-19]. Stereotaxic procedure is supposed to reduce the 
usual drawbacks when manual RF, tamponade and X-ray exposure notably [10-20]. New robotic technologies seem to be as effective as manual RF $[6,13,20]$. Despite of ongoing RMNS improvement in order to enhance remote navigation with fast computing hardware and new motion controllers, factors influencing PVI using RMNS are not well understood [13]. Consequently, the assessment of both strengths and weaknesses of the RMNS regarding AF ablation is clinically relevant.

The aim of this study was to itemize clinical and anatomical factors influencing stereotaxic PVI duration when AF ablation.

\section{Methods}

\section{Study population}

The current trial was an observational, prospective, and blinded endpoint-assessment trial. This monocentric trial included 102 consecutive patients hospitalized for a first procedure of $\mathrm{AF}$ ablation in the electrophysiology department of the University Hospital of Saint-Étienne (France) from February 2015 to April 2016. All patients underwent AF ablation by a single experienced operator accustomed to stereotaxic procedures.

Inclusion criteria were: first ablation procedure due to symptomatic and drug refractory AF, be over 18 years old and collection of an oral consent. Pregnant women were excluded as patients with left atrial appendage (LAA) thrombus. The local ethics review committee approved the study.

The following data were prospectively collected: demographic patients data, comorbidities, AF background and AF therapeutic management.

\section{Pre procedure imaging}

Conventional transthoracic echocardiography (TTE) and transesophageal echocardiography (TEE) were systematically performed 24-72 hours before the ablation procedure with a commercially available system (Vivid E9, GE Healthcare, France). During TEE study, LAA was carefully analyzed to detect left atrial thrombi and spontaneous echo contrast [21].

Cardiac computed tomography (CCT) was performed using 256-slice (Somatom Definition Flash, Siemens Medical Solutions, Erlangen, Germany) scanner technologies with similar protocols in patients in a supine position during suspended end-expiration, $24 \mathrm{~h}$ to $72 \mathrm{~h}$ prior of the AF ablation procedure. Post-processing of cardiac computed tomographic images was performed on a dedicated advanced image processing workstation (Aquarius intuition, Tera recon, Foster City, CA). Reconstructed cardiac computed tomographic images were reviewed and interpreted by an experienced independent investigator blinded to clinical and echocardiographic data. The anatomy of PVs, LAA and left atrium
(LA) ridge were assessed. CCT procedure and CT-scan data are reported in the supplementary appendix.

\section{Ablation procedure}

The procedure is detailed in the supplementary appendix. LA mapping was performed using CartoÒ 3 System (Carto® 3 system, Bio sense Webster, CA), an electromagnetic system allowing realtime Advanced Catheter Location ${ }^{\mathrm{TM}}$ and visualization of both ablation and circular mapping catheters (NaviStar $₫$ and Lasso catheters ${ }^{\circledR}$ ). Once the map was completed, 3D computed tomography scan was performed in order to optimize LA reconstruction.

The RMNS (Niobe ${ }^{\mathrm{TM}}$ EPOCH, Stereotaxis Inc., St Louis, MO) employs a steerable magnetic field remotely guiding a flexible catheter [6, 9-13]. Two giant computer-controlled 1.8-ton magnets are positioned at opposite sides of the fluoroscopy table. A magnetic field of 0.08 to 0.1 Tesla is generated allowing a 3D navigation thanks to three small magnets incorporated in parallel in the RF catheter tip. The magnetic field is applied to a theoretical cardiac volume of $20 \mathrm{~cm} \times 20 \mathrm{~cm}$. Catheter movements depend on direction changes of the two magnets in relation to each other. A computerized motor drive system (Cardiodrive $\AA$, Stereotaxis Inc., St Louis, MO) advances or retracts the catheters, whilst its spatial orientation requires a computerized work station (Navigant ${ }^{\circledR} 2.1$, Stereotaxis Inc., St Louis, MO). A constant application of the magnetic field maintains contact between the catheter tip and endocardial tissue throughout the cardiac cycle. The new generation RMNS results in faster control of the catheter, leading to potentially reduced navigation duration [22].

\section{Procedure and fluoroscopy parameters}

Skin to skin total duration was recorded for all patients. The following parameters were also recorded: X-ray duration (sec), $\mathrm{X}$-ray (Gy) and indexed X-ray (Gy x $\mathrm{cm}^{2}$ ) procedure time. These parameters were divided in different periods: setting up, mapping and ablation period (including left and right PVI).

\section{Statistical analysis}

Continuous variables were presented as mean $\pm \mathrm{SD}$, or median+IQR as appropriate. Categorical variables were expressed as percentage. Linear uni and multi-variate models were generated to predict procedure duration. Characteristics of each model were given at the regression parameter for each variable (b), with its 95\% confidence intervals and p-value. The multiple linear regression model was built in a backward stepwise manner, selecting theoretically impacting covariates (defined by $\mathrm{p}<0.05$ in the univariate analysis) to predict procedure times and X-ray patient exposure, to maximize the goodness of fit expressed as $\mathrm{R}^{2}$. All analyses were performed using R (R Foundation for Statistical Computing, Vienna, Austria, http://www.R-project.org). 


\section{Results}

\section{Patients}

Population data are summarized in (Table 1). One hundred and two consecutive patients were prospectively included, divided into 63 paroxysmal AF (62\%) and 39 persistent and long-standing AF
(38\%). The population characteristics were as follows: mean age of $59 \pm 12$ years old, $77 \%$ of male, a body mass index of $27 \pm 4.5 \mathrm{~kg} / \mathrm{m} 2$, mean CHA2DS2VASc of 1 [1-2], anticoagulated with non-vitamin $\mathrm{K}$ oral anticoagulant (81\%) and without cardiomyopathy. Mean LA surface was $23 \pm 6.5 \mathrm{~cm} 2$ and LAA normocontractility was mostly assessed.

Table 1: Patient characteristics.

\begin{tabular}{|c|c|c|c|}
\hline & $\begin{array}{c}\text { Total } \\
(n=102)\end{array}$ & Paroxysmal AF $(n=63)$ & Persistent AF $(n=39)$ \\
\hline \multicolumn{4}{|c|}{ Co-morbidities } \\
\hline Male & $79(77.5 \%)$ & $48(76.2 \%)$ & $31(79.5 \%)$ \\
\hline Age & $58.9 \pm 12$ & $58.1 \pm 12.7$ & $60.2 \pm 10.2$ \\
\hline BMI $(\mathrm{kg} / \mathrm{m} 2)$ & $27.2 \pm 4.4$ & $26.8 \pm 4.4$ & $27.7 \pm 4.3$ \\
\hline Dyslipidaemia & $27(26.5 \%)$ & $16(25.4 \%)$ & $11(28.2 \%)$ \\
\hline Coronary heredity & $14(13.7 \%)$ & $7(11.1 \%)$ & $7(17.9 \%)$ \\
\hline Tobacco use & $45(44.1 \%)$ & $26(41.3 \%)$ & $19(48.7 \%)$ \\
\hline Hypertension & $47(46.1 \%)$ & $27(26.5 \%)$ & $20(51.3 \%)$ \\
\hline Diabetes mellitus & $8(7.8 \%)$ & $4(6.3 \%)$ & $4(10.3 \%)$ \\
\hline \multicolumn{4}{|c|}{ Thomboembolism risk prevention } \\
\hline Stroke & $3(2.9 \%)$ & $1(1.6 \%)$ & $2(5.1 \%)$ \\
\hline CHA2DS2VASc & $1.3 \pm 1.3$ & $1.2 \pm 1.3$ & $1.4 \pm 1.2$ \\
\hline NOAC anticoagulation & $83(81.4 \%)$ & $56(88.9 \%)$ & $27(69.2 \%)$ \\
\hline Previous flutter ablation & $14(13.7 \%)$ & $8(12.7 \%)$ & $6(15.4 \%)$ \\
\hline \multicolumn{4}{|c|}{ Echocardiographic characteristics } \\
\hline LVEF & $58.8 \pm 8.5$ & $60.3 \pm 7.0$ & $56.5 \pm 10.2$ \\
\hline Mitral regurgitation & $83(81.4 \%)$ & $50(79.4 \%)$ & $33(84.6 \%)$ \\
\hline Indexed left atrium volume $\left(\mathrm{mL} / \mathrm{m}^{2}\right)$ & $44.4 \pm 18.6$ & $42.1 \pm 18.8$ & $48.3 \pm 17.9$ \\
\hline Left atrium surface $\left(\mathrm{cm}^{2}\right)$ & $22.8 \pm 6.4$ & $21.7 \pm 6.0$ & $24.4 \pm 6.6$ \\
\hline Right atrium surface $\left(\mathrm{cm}^{2}\right)$ & $20.1 \pm 6.3$ & $19.5 \pm 6.1$ & $21.0 \pm 6.7$ \\
\hline LAA normocontractility & $77(75.5 \%)$ & $52(82.5 \%)$ & $25(64.1 \%)$ \\
\hline \multicolumn{4}{|c|}{ CT scan characteristics } \\
\hline Indexed left atrium volume $\left(\mathrm{mL} / \mathrm{m}^{2}\right)$ & $52.8 \pm 15.3$ & $48.3 \pm 13.6$ & $60.5 \pm 15.0$ \\
\hline Left atrium surface $\left(\mathrm{cm}^{2}\right)$ & $24.4 \pm 6.0$ & $23.2 \pm 4.9$ & $26.4 \pm 7.0$ \\
\hline
\end{tabular}

Continuous variables are presented as mean $\pm \mathrm{SD}$. Categorical variables are expressed as number (percentage). $\mathrm{AF}=\mathrm{atrial}$ fibrillation; $\mathrm{BMI}=$ body mass index; LAA=left atrial appendage; $\mathrm{LVEF}=$ left ventricular ejection fraction; NOAC=non-vitamin $\mathrm{K}$ antagonist oral anticoagulant; sAoVTI=sub aortic velocity time integral.

\section{Procedure features}

Procedure parameters are summarized in (Table 2). A 100\% acute PV isolation success was reported. Mean ablation procedure time was $97 \pm 33$ minutes with a mean RF time of $66 \pm 31$ minutes. Total X-ray duration was $13.4 \pm 7.9$ minutes. Fluoroscopic use was mainly related to the setting up period (58\%), compared to $23 \%$ of ablation procedure duration. Ablation and total duration are not different whether paroxysmal or persistent AF. Three acute complications occurred: a pericardial effusion without tamponade and two medically-treated inguinal haematomas.

Table 2: Procedure parameters.

\begin{tabular}{|c|c|c|c|c|c|c|}
\hline & \multicolumn{3}{|c|}{ Procedure Duration (min) } & \multicolumn{3}{c|}{ Fluoroscopy Duration (min) } \\
\hline & Paroxysmal AF & Total & Persistent AF & Paroxysmal AF & Total & Persistent AF \\
\hline Ablation period & $63.4 \pm 28.2$ & $65.6 \pm 31.4$ & $69.0 \pm 36.1$ & $3.0 \pm 2.4$ & $2.9 \pm 1.8$ & $2.9 \pm 3.1$ \\
\hline Whole procedure & $97.1 \pm 29.8$ & $97.2 \pm 32.9$ & $97.4 \pm 37.9$ & $14.1 \pm 9.5$ & $13.4 \pm 7.9$ & $12.4 \pm 4.2$ \\
\hline
\end{tabular}

Continuous variables are presented as mean $\pm \mathrm{SD}$. $\mathrm{AF}=$ atrial fibrillation. 


\section{Univariate analysis}

Clinical characteristics impacting the ablation duration No co-morbidity was associated with an increase of the AF ablation procedure or X-ray exposure duration (Table 3). On the other hand, persistent versus paroxysmal $\mathrm{AF}(\mathrm{p}<0.05)$, and previous flutter ablation $(\mathrm{p}<0.01)$ were both risk factors associated with a with procedure duration. The left ventricular function was not associated with a change in ablation procedure duration parameters (Table 3 ): neither LVEF ( $p=0.2$ ) nor sAoTVI $(=0.6)$. Furthermore, mitral valve disease, whether mitral regurgitation $(p=0.9)$ or stenosis $(p=0.4)$, was not correlated to longer procedures.

Table 3: Impact of clinical, hemodynamic and anatomical characteristics on the procedure duration.

\begin{tabular}{|c|c|c|c|}
\hline & \multicolumn{3}{|c|}{ Procedure duration } \\
\hline & $\beta$ & $95 \% \mathrm{CI}$ & $\mathrm{p}$ \\
\hline \multicolumn{4}{|c|}{ Co-morbidities } \\
\hline Age & 0.13 & {$[-0.4 ; 0.7]$} & 0.6 \\
\hline Diabetes mellitus & -13 & {$[-37 ; 11]$} & 0.2 \\
\hline Vascular disease & 20 & {$[-14 ; 45]$} & 0.1 \\
\hline BMI & 0.6 & {$[-0.9 ; 2.1]$} & 0.4 \\
\hline \multicolumn{4}{|c|}{ Atrial Fibrillation Characteristics } \\
\hline Persistent AF & 14 & {$[0,9 ; 27]$} & $<0.05$ \\
\hline Previous flutter ablation & 34 & {$[17 ; 52]$} & $<0.01$ \\
\hline \multicolumn{4}{|c|}{ Haemodynamic characteristics } \\
\hline LVEF & -0.5 & {$[-1.3 ; 0.3]$} & 0.2 \\
\hline Average mitral gradient & 3.8 & {$[-5.1 ; 12.6]$} & 0.4 \\
\hline Mitral regurgitation & -0.8 & {$[-17 ; 16]$} & 0.9 \\
\hline \multicolumn{4}{|c|}{ Anatomical characteristics } \\
\hline Atrial volume & 0.2 & {$[0.1 ; 0.5]$} & $<0.05$ \\
\hline Atrial area & 1.4 & {$[0.3 ; 2.5]$} & $<0.05$ \\
\hline Ridge width & -10.7 & {$[-19,0 ;-2,4]$} & 0.01 \\
\hline LSPV area & -0.13 & {$[-0.4 ;-0,17]$} & 0.4 \\
\hline LSPV eccentricity & -87 & {$[-323 ;-198]$} & 0.5 \\
\hline LIPV area & -0.25 & {$[-0.66 ;-0.16]$} & 0.2 \\
\hline LIPV eccentricity & 299.6 & {$[86.2 ; 513.0]$} & $<0.01$ \\
\hline
\end{tabular}

Univariate analysis. AF=atrial fibrillation; $\mathrm{BMI}=$ body mass index; LVEF=left ventricular ejection fraction; LIPV=left inferior pulmonary vein; LSPV=left superior pulmonary vein.

Anatomical characteristics impacting the ablation duration LA dilation, assessed by LA area $(\mathrm{p}<0.05)$ and LA volume $(\mathrm{p}<0.05)$, was associated to an increased procedure duration (Table 3 ). This association was found during the mapping poeriod of the procedure $(\mathrm{p}<0.01)$, but not for the setting up and ablation ones (Table 1), supplementary appendix). On the other side, wider LAA ridge was correlated with a shorter fluoroscopy duration $(\mathrm{p}=0.01)$. Smaller LIPV surface area $(p<0.01)$ and higher LIPV eccentricity $(p<0.01)$ were correlated with longer RF duration (Table 2), supplementary appendix).

\section{Multivariate analysis}

Through multivariate linear regression analysis with relevant clinical and echocardiographic features, a previous flutter ablation $(p<0.01)$ and persistent $A F(p=0.03)$ were found to be independently associated with total procedure duration. Furthermore, left LA fluoroscopy duration was independently influenced by LIPV eccentricity $(\mathrm{p}<0.05)$ and LA ridge width $(\mathrm{p}=0.05)$.

\section{Discussion}

\section{Major findings}

This prospective observational study suggests that no comorbidity and cardiomyopathy was associated to longer stereotaxic PVI procedures. LA dilation was correlated with increased setting up but not ablation duration. Persistent AF and previous atrial flutter are independently associated to an increased procedure duration. Only LA ridge width and LIPV anatomy influenced significantly and independently the ablation duration.

\section{Stereotaxic procedure duration}

Due to the improving indications of $\mathrm{AF}$ ablation, all dedicated EP departments deal with an increased daily ablation procedure. 
Thereby, different challenges appear: [1] decrease procedure duration leading to reduce physician fatigue and X-ray exposure and [2] optimize the management of consecutive daily procedures. As an indicator shown in (Figure 1), the procedure duration seems to be significantly shorter in the current study using stereotaxic system, compared to cry balloon and manual RF ablation in FIRE and ICE trial [23]. Indeed, manual RF ablation seems to approximately 50\% longer than stereotaxic procedure, while fluoroscopy duration is $75 \%$ longer with cry balloon and 33\% longer with manual RF than stereotaxic ablation. In addition to a shorter procedure, stereotaxic ablation reduces the operator tiredness and increases its accuracy by allowing a seated and comfortable procedure. However, it is important to keep in mind that all ablations were performed with an experienced operator, after a usual learning curve.

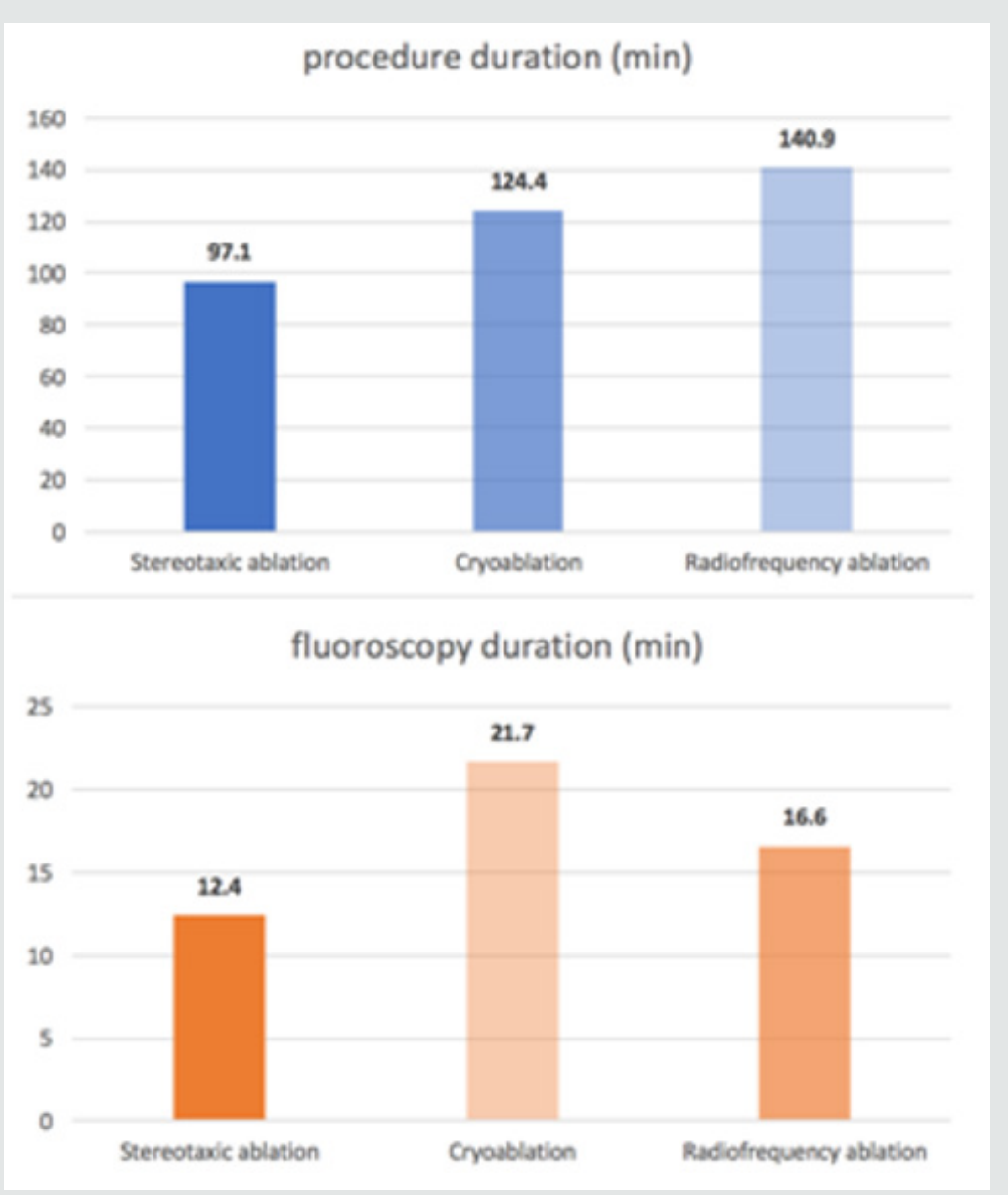

Figure 1: Procedure and fluoroscopy duration with stereotaxic ablation compared to cryoballoon and radiofrequency ablation in FIRE and ICE trial [23].

Factors influencing procedure duration (Figure 2) Clinical characteristics impacting the ablation duration. Age, sex, diabetes mellitus, vascular disease and obesity are not correlated with an increased manual RF procedure duration [24], which is consistent with our trial. Our study was the first highlighting the lack of association between body weight and procedure duration. In addition, $\mathrm{AF}$ radiofrequency seems to be safe despite of overweight: there is not a higher hemorrhagic and infectious complications incidence reported [25]. Yet several AF risk factors such as obesity and sleep apnea, seems to be important to maintain a sinus rhythm after AF ablation [26]. Persistent AF was associated to a longer PVI procedure, suggesting the presence of more severe LA architectural abnormalities when persistent AF. Indeed, persistent AF seems to be associated to a much more severe atrial cardiomyopathy compared to paroxysmal AF [27]. Furthermore, previous ablation of atrial flutter RF was an independent risk factor of longer ablation procedure. The interrelationship between $\mathrm{AF}$ and atrial flutter is still unclear [28]. But this finding suggests that patients with previous atrial flutter get a complex atrial cardiomyopathy including fibrosis [24] and atrial dilation. It could lead to a longer and less efficient IVP procedure [29]. 


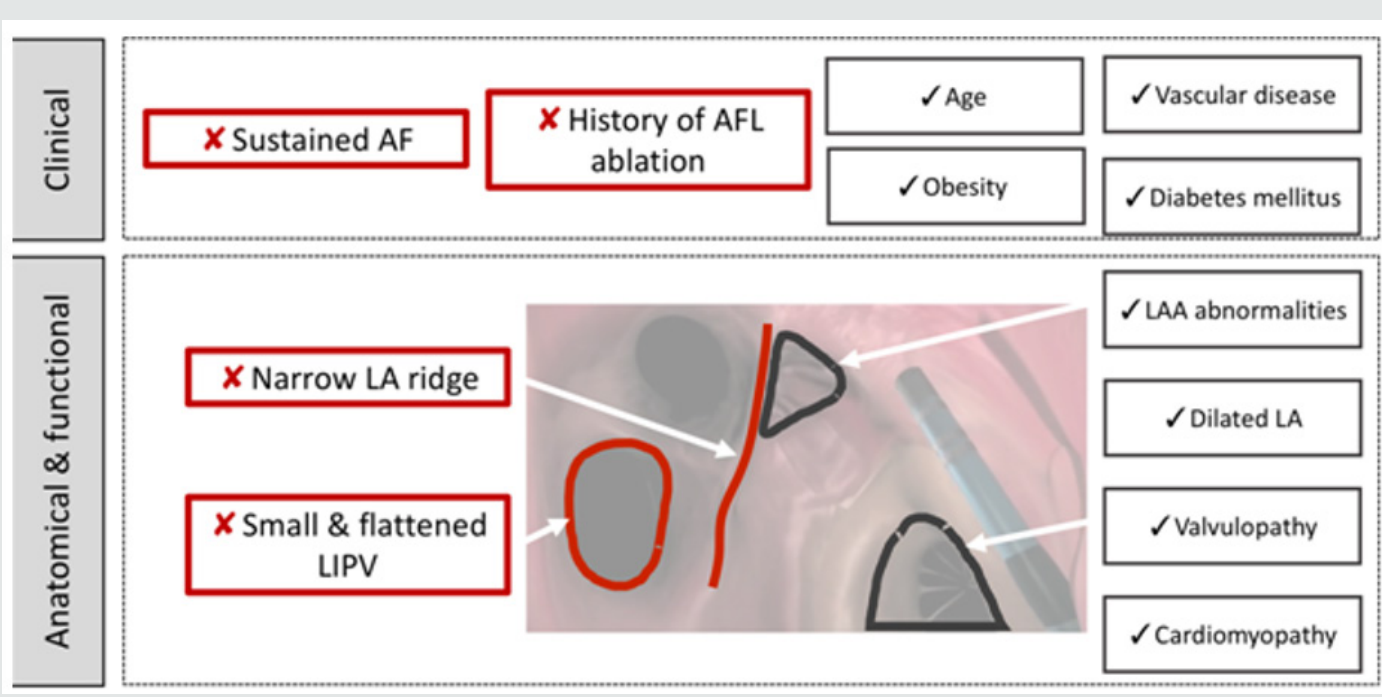

Figure 2: Central figure: Clinical, hemodynamic and anatomical factors influencing the stereotaxic AF ablation duration.

This figure summarizes results of the univariate analysis. Image corresponds to an endocavity view of the left atrium with a septal view. $\mathrm{AF}=$ atrial fibrillation; $\mathrm{AFL}=$ atrial flutter; $\mathrm{LIPV}=$ left inferior pulmonary vein; $\mathrm{LA}=$ left atrium; $\mathrm{LAA}=\mathrm{left}$ atrial appendage.

Hemodynamic characteristics impacting the ablation duration neither systolic left ventricular dysfunction nor mitral disease were correlated to a longer procedure. Since $50 \%$ of AF patients get heart failure and $25 \%$ of heart failure patients get $\mathrm{AF}$ [30], the efficacy and safety of the AF ablation has to be proved. Indeed, antiarrhythmic drugs fail AF patients with heart failure [31], and $\mathrm{AF}$ ablation could be a therapeutic key, as suggested by CASTLE-AF trial [32]. In this study, catheter ablation decreased hospitalization rate and mortality, increased left ventricular function, over midterm follow up [33]. The stereotaxic ablation seems to be an interesting strategy when heart failure, regarding the safety of the procedure and the absence of LV dysfunction and LA dilation impact in procedure duration.

Left atrial architectural features impacting the ablation duration In our study, LA dilation led to a longer procedure, with an increased mapping duration, yet no effect on stereotaxic ablation duration was reported. Stereotaxic procedure seems to overcome anatomical difficulties such as LA dilation, preventing technical difficulties because of an efficient navigation.

On the other side, a narrow ridge was a predictor of longer ablation using RMNS. The left lateral ridge is known as a uniform width or muscular thickness being narrower and thicker at the antero-superior level [34]. This area acts as a fibrillary process due to the presence of the vein of Marshall, an electrical gap between left PV and LA [35]. This area constitutes a preferential zone of PVs reconnection [36]. Manual RF procedures often fail to complete ablation line in LA ridge [37]. This study also pointed out a tough ablation in this area despite stereotaxic accurate navigation.

\section{Clinical implications}

$\mathrm{AF}$ catheter ablation is an efficient treatment to achieve a rhythm control strategy, regarding recent guidelines [8]. But this procedure is still challenging and needs to be more efficient and safer. RMNS may be an interesting way to achieve this goal. In this study, all procedures allow an acute PV isolation, associated to a short procedure and fluoroscopy time. Furthermore, RMNS allows an efficient navigation during PVI, regardless LA dilation and left ventricular dysfunction.

But RMNS accuracy could be improved. The Stereotaxis Magnetic Navigation System allows precise navigation with a spatial resolution of 1 degree of omni-directional deflection and $1 \mathrm{~mm}$ for catheter advancement and retraction, as opposed to the manual catheter manipulation and catheter movements which highly depends on the operator. RMNS leads to a stable cathetertissue contact during cardiac motion, unlike manual RF ablation [38]. This study suggests that a narrow LA ridge and a small and flattened LIPV increase overall procedure time. The knowledge of these anatomical limitations ta achieve IVP may help the engineers to work on RMNS improvements.

\section{Study limitations}

The major limitation of this observational study was its lack of randomization. However, this study included consecutive patients, prospectively, in order to prevent bias analysis. In addition, this monocentric trial was based on IVP procedures performed by a single operator. It avoids the inter-observer variability but limits the exptrapolability of the data. Finally, a new trial should be 
designed to test LA anatomical predictors of AF recurrences and PVs reconnection with RMNS compared to manual RF and cry balloon procedures.

\section{Conclusion}

Our study proposed to highlight predictors of stereotaxic procedure duration. Both narrow LA ridge, small and flattened left inferior PV are independently correlated with increased procedure duration. But no co-morbidity and cardiomyopathy were linked to a procedure change.

\section{Supplementary Appendix}

\section{References}

1. Reynolds MR, Zimetbaum P, Josephson ME, Ellis E, Danilov T, et al. (2009) Cost-effectiveness of radiofrequency catheter ablation compared with antiarrhythmic drug therapy for paroxysmal atrial fibrillation. Circulation Arrhythmia and Electrophysiology 2(4): 362-369.

2. January CT, Wann LS, Alpert JS, Calkins H, Cigarroa JE, et al. (2014) 2014 AHA/ACC/HRS Guideline for the Management of Patients With Atrial Fibrillation: Executive Summary. Journal of the American College of Cardiology 130(23): 2071-2104

3. Kirchhof P, Benussi S, Kotecha D, Ahlsson A, Atar D, et al. (2016) 2016 ESC Guidelines for the management of atrial fibrillation developed in collaboration with EACTS. European Heart Journal 37(38): 2893-962.

4. Macle L, Weerasooriya R, Jais P, Scavee C, Raybaud F, et al. (2003) Radiation exposure during radiofrequency catheter ablation for atrial fibrillation. Pacing and Clinical Electrophysiology : PACE 26(1p2): 288291.

5. Venneri L, Rossi F, Botto N, Andreassi MG, Salcone N, et al. (2009) Cancer risk from professional exposure in staff working in cardiac catheterization laboratory: insights from the National Research Council's Biological Effects of Ionizing Radiation VII Report. American Heart Journal 157(1): 118-124.

6. Link MS, Haïssaguerre M, Natale A (2016) Ablation of Atrial Fibrillation: Patient Selection, Periprocedural Anticoagulation, Techniques, and Preventive Measures After Ablation. Circulation 134(4): 339-352.

7. Terasawa T, Balk EM, Chung M, Garlitski AC, Alsheikh-Ali AA, et al. (2009) Systematic review: comparative effectiveness of radiofrequency catheter ablation for atrial fibrillation. Annals of Internal Medicine 151(3): 191-202.

8. Kirchhof P, Benussi S, Kotecha D, Ahlsson A, Atar D, et al. (2016) 2016 ESC Guidelines for the management of atrial fibrillation developed in collaboration with EACTS: The Task Force for the management of atrial fibrillation of the European Society of Cardiology (ESC)Developed with the special contribution of the Europea. European Heart Journal 37(38): 2893-2962.

9. Calkins H, Kuck KH, Cappato R, Brugada J, Camm AJ, Chen S-A, et al. (2012) 2012 HRS/EHRA/ECAS expert consensus statement on catheter and surgical ablation of atrial fibrillation: recommendations for patient selection, procedural techniques, patient management and follow-up, definitions, endpoints, and research trial design: a re. Heart Rhythm: The Official Journal of the Heart Rhythm Society 9(4): 632-696.e21.

10. Faddis MN, Blume W, Finney J, Hall A, Rauch J, Sell J, et al. (2002) Novel, magnetically guided catheter for endocardial mapping and radiofrequency catheter ablation. Circulation 106(23): 2980-2985.

11. Greenberg S, Blume W, Faddis M, Finney J, Hall A, et al. (2006) Remote controlled magnetically guided pulmonary vein isolation in canines. Heart Rhythm 3(1): 71-76.
12. Faddis MN, Chen J, Osborn J, Talcott M, Cain ME, et al. (2003) Magnetic guidance system for cardiac electrophysiology: a prospective trial of safety and efficacy in humans. Journal of the American College of Cardiology 42(11): 1952-1958.

13. Weiss JP, May HT, Bair TL, Crandall BG, Cutler MJ, et al. (2016) A Comparison of Remote Magnetic Irrigated Tip Ablation versus Manual Catheter Irrigated Tip Catheter Ablation With and Without Force Sensing Feedback. Journal of Cardiovascular Electrophysiology 27 (Suppl 1): S510.

14. Adragão PP, Cavaco D, Ferreira AM, Costa FM, Parreira L, et al. (2016) Safety and Long-Term Outcomes of Catheter Ablation of Atrial Fibrillation Using Magnetic Navigation versus Manual Conventional Ablation: A Propensity-Score Analysis. Journal of Cardiovascular Electrophysiology 27 (Suppl 1): S11-16.

15. Jin QI, Pehrson S, Jacobsen PK, Chen XU (2016) Efficacy and Safety of Atrial Fibrillation Ablation Using Remote Magnetic Navigation: Experience from 1,006 Procedures. Journal of Cardiovascular Electrophysiology 27(Suppl 1): S23-28.

16. Ernst S, Ouyang F, Linder C, Hertting K, Stahl F, Chun J, et al.(2004) Initial experience with remote catheter ablation using a novel magnetic navigation system: magnetic remote catheter ablation. Circulation 109(12): $1472-1475$

17. Pappone C, Vicedomini G, Manguso F, Gugliotta F, Mazzone P, et al. (2006) Robotic magnetic navigation for atrial fibrillation ablation. Journal of the American College of Cardiology 47(7): 1390-1400.

18. Bradfield J, Tung R, Mandapati R, Boyle NG, Shivkumar K, et al. (2012) Catheter ablation utilizing remote magnetic navigation: a review of applications and outcomes. Pacing and Clinical Electrophysiology : PACE 35(8): 1021-1034.

19. Shurrab M, Danon A, Lashevsky I, Kiss A, Newman D, et al. (2013) Robotically assisted ablation of atrial fibrillation: a systematic review and meta-analysis. International Journal of Cardiology 169(3):157-165.

20. Da Costa A, Lafond P, Romeyer-Bouchard C, Gate-Martinet A, Bisch L, Nadrouss A, et al. Remote magnetic navigation and arrhythmia ablation. Archives of Cardiovascular Diseases 105(8-9): 446-453.

21. Beigel R, Wunderlich NC, Ho SY, Arsanjani R, Siegel RJ, et al. (2014) The left atrial appendage: anatomy, function, and noninvasive evaluation. JACC Cardiovascular Imaging 7(12): 1251-1265.

22. Da Costa A, Guichard JB, Maillard N, Romeyer-Bouchard C, Gerbay A, et al. (2017) Substantial superiority of Niobe ES over Niobe II system in remote-controlled magnetic pulmonary vein isolation. Int J Cardiol 230: 319-323.

23. Kuck K-H, Brugada J, Fürnkranz A, Metzner A, Ouyang F, Chun KRJ, et al. (2016) Cryoballoon or Radiofrequency Ablation for Paroxysmal Atrial Fibrillation. New England Journal of Medicine NEJMoa1602014.

24. Sotomi Y, Inoue K, Ito N, Kimura R, Toyoshima Y, et al. (2013) Incidence and risk factors for very late recurrence of atrial fibrillation after radiofrequency catheter ablation. Europace 15(11): 581-586.

25. Letsas KP, Siklódy CH, Korantzopoulos P, Weber R, Bürkle G, et al. (2013) The impact of body mass index on the efficacy and safety of catheter ablation of atrial fibrillation. International Journal of Cardiology 164(1): 94-98.

26. Rajeev KP, Melissa EM, Dennis HL, Abhinav BM, Rajiv M, et al. (2014) Aggressive risk factor reduction study for atrial fibrillation and implications for the outcome of ablation: the ARREST-AF cohort study. J Am Coll Cardiol 64(21): 2222-2231.

27. Mahnkopf C, Badger TJ, Burgon NS, Daccarett M, Haslam TS, et al (2010) Evaluation of the left atrial substrate in patients with lone atrial fibrillation using delayed-enhanced MRI: implications for disease progression and response to catheter ablation. Heart Rhythm $7(10): 1475-1481$ 
28. Waldo AL (2005) The Interrelationship Between Atrial Fibrillation and Atrial Flutter. Progress in Cardiovascular Diseases 48(1): 41-56.

29. Nattel S, Harada M (2014) Atrial remodeling and atrial fibrillation: recent advances and translational perspectives. Journal of the American College of Cardiology 63(22): 2335-2345.

30. Nieuwlaat R, Eurlings LW, Cleland JG, Cobbe SM, Vardas PE, et al. (2009) Atrial fibrillation and heart failure in cardiology practice: reciprocal impact and combined management from the perspective of atrial fibrillation: results of the Euro Heart Survey on atrial fibrillation. Journal of the American College of Cardiology 53(18): 1690-1698.

31. Roy D, Talajic M, Nattel S, Wyse DG, Dorian P, et al. (2008) Rhythm control versus rate control for atrial fibrillation and heart failure. The New England Journal of Medicine 358:2667-2677.

32. Marrouche NF, Brachmann J, Andresen D, Siebels J, Boersma L, et al. (2018) Catheter Ablation for Atrial Fibrillation with Heart Failure. N Engl J Med 378(5): 417-427.

33. Al Halabi S, Qintar M, Hussein A, Alraies MC, Jones DG, et al. (2015) Catheter Ablation for Atrial Fibrillation in Heart Failure Patients: A Meta-Analysis of Randomized Controlled Trials. JACC Clinical Electrophysiology 1(3): 200-209.
34. Macedo PG, Kapa S, Mears JA, Fratianni A, Asirvatham SJ, et al. (2010) Correlative anatomy for the electrophysiologist: ablation for atrial fibrillation. Part I: pulmonary vein ostia, superior vena cava, vein of Marshall. Journal of Cardiovascular Electrophysiology 21(6): 721-730.

35. Rodríguez-Mañero M, Schurmann P, Valderrábano M (2016) Ligament and Vein of Marshall. A therapeutic opportunity in atrial fibrillation. Heart Rhythm 13(2):593-601.

36. Rajappan K, Kistler PM, Earley MJ, Thomas G, Izquierdo M, et al. (2008) Acute and chronic pulmonary vein reconnection after atrial fibrillation ablation: a prospective characterization of anatomical sites. Pacing and Clinical Electrophysiology : PACE 31(12): 15981605.

37. Kuck K-H, Brugada J, Fürnkranz A, Metzner A, Ouyang F, et al. (2016) Cryoballoon or Radiofrequency Ablation for Paroxysmal Atrial Fibrillation. New England Journal of Medicine 37(38): 2858-2865.

38. Bhaskaran A, Barry MAT, Al Raisi SI, Chik W, Nguyen DT, et al. (2015) Magnetic guidance versus manual control: comparison of radiofrequency lesion dimensions and evaluation of the effect of heart wall motion in a myocardial phantom. Journal of Interventional Cardiac Electrophysiology : An International Journal of Arrhythmias and Pacing 44:1-8.

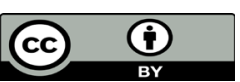

This work is licensed under Creative Commons Attribution 4.0 License

To Submit Your Article Click Here:

Submit Article

DOI: $10.32474 /$ ACR.2021.03.000164

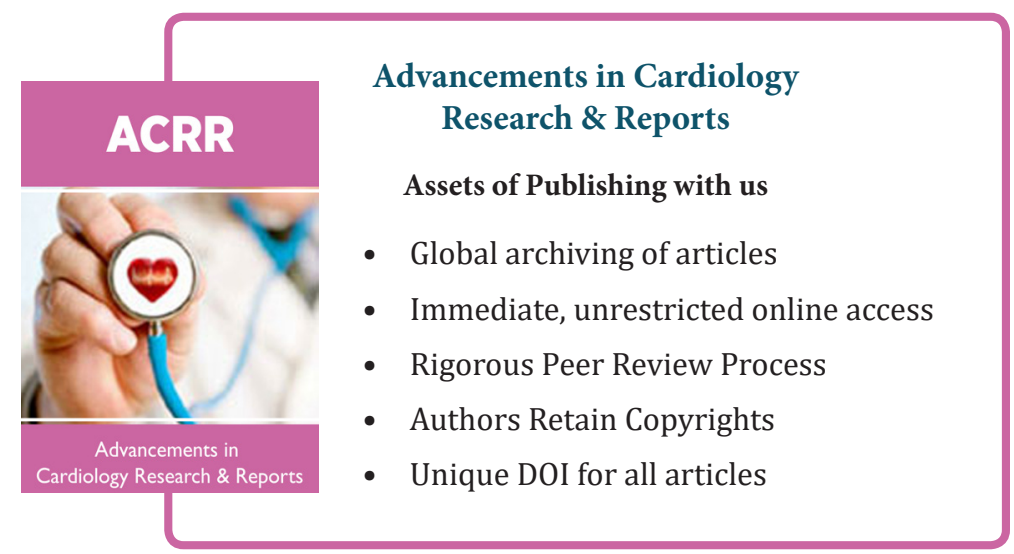

